\title{
Short-Duration X-ray Transients Observed With WATCH on Granat: Are Some of Them Related to Stellar Flares?
}

\author{
Alberto J. Castro-Tirado ${ }^{1}$, Søren Brandt ${ }^{2}$, Niels Lund ${ }^{2}$, Igor Lapshov ${ }^{3}$, \\ Rashid Sunyaev ${ }^{3}$ \\ 1 Laboratorio de Astrofísica Espacial y Física Fundamental (LAEFF-INTA), \\ P.O. Box 50727, E-28080 Madrid, Spain \\ 2 Danish Space Research Institute, Gl. Lundtoftevej 7, DK-2800 Lyngby, Denmark \\ 3 Space Research Institute, Academy of Sciences, Profsoyuznaya 84/32, \\ 117810 Moscow, Russia
}

\begin{abstract}
During 1990-92, the WATCH all-sky X-ray monitor on GRANAT has discovered 6 short-duration X-ray transients. We discuss their possible relationship to peculiar stars. Only one source, GRS $1100-77$ seems to be related to a T Tauri star.
\end{abstract}

\section{Introduction}

Amongst the many kinds of sources in the variable X-ray sky, X-ray transients have been observed since the first experiments in the late 60s. According to their duration, it is possible to distinguish between long-duration X-ray transients (lasting from weeks to few months) and short-duration X-ray transients (lasting from hours to very few days).

Long-duration X-ray transients are mainly related to Be-neutron star systems (Be/X-ray Transients) and to soft X-ray transients (a subclass of the low-mass $\mathrm{X}$-ray binaries). The latter include the best black-hole candidates found so far.

Not too much is known with respect to most short-duration X-ray transients. The main reason is the lack of counterparts at other wavelengths for many of them. One subclass, the so-called fast X-ray transients, have been observed with many detectors since the launching of the Vela satellites (Heise et al. 1975, Cooke 1976). Durations range from seconds to less than few hours. Normally, they have been seen only once, and they never have been detected in quiescence, implying high peak-to-quiescent flux ratios $\left(10^{2}-10^{3}\right)$ (Ambruster et al. 1983). Spectral characteristics vary substantially, from a hard spectrum in MX 2346-65 $(k T \sim$ $20 \mathrm{keV}$; Rappaport et al. 1976) to soft spectra with blackbody temperatures from $k T=0.87$ to $2.3 \mathrm{keV}$ (Swank et al. 1977). In the Ariel $V$ database, 27 sources were discovered (Pye \& McHardy 1983), and 10 more were detected in the HEAO $1 \mathrm{~A}-1$ all sky survey (Ambruster \& Wood, 1986), implying a fast transient all-sky event rate of $1500 \mathrm{yr}^{-1}$ for fluxes $F_{\mathrm{X}} \geq 310^{-10} \mathrm{erg} \mathrm{cm}^{-2} \mathrm{~s}^{-1}$ in the (2-10 keV) energy band. In the HEAO $1 A-2$ survey, 52 events were found (Connors 1988), but 37 of them were associated with 4 of the brightest X-ray sources in the LMC. Due to the large difference of observational characteristics, 
it seems that these events are caused by more than one physical mechanism. In several cases, there have been tentative optical identifications on the basis of known sources in the transient error boxes. One suggestion has been that many of the fast $\mathrm{X}$-ray transients are related with stellar flares originated in active coronal sources, like RS CVn binaries or dMe-dKe flare stars.

RS CVn systems peak luminosities are usually $L_{\mathrm{X}} \sim 10^{32} \mathrm{erg} \mathrm{s}^{-1}$. The hardest flare yet observed was for the system HR 1099, on $17 \mathrm{Feb} 1980$. It was detected with HEAO 2 at energies up to $20 \mathrm{keV}$ (Agrawal \& Vaidya 1988). The most energetic X-ray flare was observed with Ginga from UX Ari. Its decay time was quite long $(\sim 0.5 \mathrm{~d})$.

$\mathrm{X}$-ray flares can be also observed from dMe-dKe stars. In the Ariel $V$ sky survey, Rao \& Vahia (1984) suggested $7 \mathrm{dMe}$ stars as responsible for X-ray flares that reached peak luminosities $L_{\mathrm{X}}<10^{32} \mathrm{erg} \mathrm{s}^{-1}(2-18 \mathrm{keV})$. AT Mic is the dMe star with the largest number of recorded events (4). For a flare observed with EXOSAT from YY Gem on 15 Nov 1984, Pallavicini et al. (1990) estimated a total flare energy $E_{\mathrm{X}}=10^{34} \mathrm{erg}(0.005-2 \mathrm{keV})$, and a decay time $t_{\mathrm{d}}=65 \mathrm{~min}$ (one of the longest decay times ever mesured for such a flares). The longest flare ever reported was observed for more than $2 \mathrm{~h}$ for the X-ray source EXO 0408307134.7 (Van der Woerd et al. 1989). X-ray flares have also been observed from Algol-type binaries (Schnopper et al. 1976), W Uma systems and young stars. See Linsky (1991) for a comprenhensive review. Theoreticians have suggested other sources like dwarf novae (Stern et al. 1981) or extreme type I X-ray bursts (Lewin \& Joss 1981) as origin of some fast X-ray transients.

\section{Observations by WATCH during 1990-92}

The WATCH instrument was the all-sky X-ray monitor onboard the GRANAT satellite, launched on 1 Dec 1989. It consisted of four identical units, mounted in a tetrahedral configuration covering the whole sky. Each instrument had an effective area of $\sim 30 \mathrm{~cm}^{2}$. It was based on the rotation modulation collimator principle (Lund 1985). The two energy ranges are approximately 8-15 and 15$100 \mathrm{keV}$. Usually, the uncertainty for the location of a new and short-duration source was 1 degree in radius (3- $\sigma$ ). A summary of scientific results can be found in Castro-Tirado (1994) and Brandt (1994).

\subsection{Fast X-ray transients discovered with WATCH}

During 1990-1992, WATCH discovered 3 bright fast X-ray transients, a small number compared with the above-mentioned instruments. This was mainly due to the higher low-energy cut off for WATCH ( $\sim 8 \mathrm{keV})$, implying that only the harder events were detected. The events were immediately noticeable as an increase in the low-energy count rate (Fig. 1). No positive detections were made in the higher energy band. The observational characteristics of the events are summarized in Table 1. 
Table 1. WATCH Fast X-ray transients.

\begin{tabular}{|c|c|c|c|c|c|c|}
\hline Source & date & UT & $t(\min )$ & $\alpha(1950.0)$ & $\delta(1950.0)$ & $F_{\mathrm{X}}(\mathrm{Crab})$ \\
\hline GRS 1100-77 & 15 Jan 1992 & $13: 39$ & 90 & $10 \mathrm{~h} 59 \mathrm{~m} .9$ & -77.1 & 0.80 \\
GRS 2037-40 & 23 Sep 1992 & $04: 35$ & 110 & $20 \mathrm{~h} 37 \mathrm{~m} .9$ & -39.8 & 0.75 \\
GRS 2220-15 & 19 Sep 1990 & $03: 32$ & 240 & $22 \mathrm{~h} 20 \mathrm{~m} .4$ & -14.9 & 2.10 \\
\hline
\end{tabular}

Note: The UT time refers to the beginning of the event. $t$ is the duration.

\subsection{Longer duration X-ray transients discovered with WATCH}

Three long events were discovered with WATCH during 1990-92, although it is likely that some others are present in the data. The sources reported here were observed to peak at a flux $F_{\mathrm{X}} \leq 0.5 \mathrm{Crab}(8-20 \mathrm{keV})$, and were discovered by analysis of the corresponding modulation pattern data. Their observational characteristics are summarized in Table 2. A fourth event, GRS 1133+54 (lasting for $\sim 1$ d on 19-20 Nov 1992) was reported elsewhere (Lapshov et al. 1992).

Table 2. WATCH Longer duration X-ray transients.

\begin{tabular}{|c|c|c|c|c|c|c|}
\hline Source & date & UT & $t(\mathrm{~d})$ & $\alpha(1950.0)$ & $\delta(1950.0)$ & $F_{\mathbf{X}}$ (Crab) \\
\hline GRS 0818-52 & 10 Oct 1990 & $14: 00$ & $\sim 1$ & $08 \mathrm{~h} 18 \mathrm{~m} .0$ & -52.0 & 0.50 \\
GRS 1148-66 & 10 Oct 1990 & $12: 00$ & $\sim 1$ & $11 \mathrm{~h} 48 \mathrm{~m} .0$ & -66.5 & 0.35 \\
GRS 1624-37 & 24 Sep 1992 & $03: 00$ & $\sim 1$ & $16 \mathrm{~h} \mathrm{24m.4}$ & -37.5 & 0.35 \\
\hline
\end{tabular}

Note: The UT time refers to the maximum flux. $t$ is the duration.

\subsection{Search for correlated X-ray flares}

We have searched for X-ray flares in the WATCH data base that could have occurred simultaneously with observed flares at other wavelengths reported elsewhere. The results were negative both for $\lambda$ Eridani (a flare was observed with ROSAT on 21 Feb 1991, Smith et al. 1993) and AU Mic (a flare was observed with EUVE on 15 July 1992, Cully et al. 1993). In the case of EV Lac, no WATCH data were available for an extraordinary optical flare that occurred on 15 Sep 1991 (Gershberg et al. 1995).

\section{Discussion}

\subsection{The fast $\mathrm{X}$-ray transients}

The three events described in 2.1 imply a rate of $\sim 5$ year $^{-1}$. If the sources of the three events are galactic, their high galactic latitude would suggest that they are nearby, although no nearby known flare stars (from the list of Pettersen, 1991) have been found in the corresponding WATCH error boxes. 
GRS 1100-77. The observations implied $L_{\mathrm{X}}=0.610^{30}(\mathrm{~d} / 1 \mathrm{pc})^{2} \mathrm{erg} \mathrm{s}^{-1}(8-$ $15 \mathrm{keV})$, or $L_{\mathrm{X}}=2.310^{30}(\mathrm{~d} / 1 \mathrm{pc})^{2} \mathrm{erg} \mathrm{s}^{-1}(2-15 \mathrm{keV})$ if we assume a Crab-like spectrum. The most interesting object inside the error box is the $\mathrm{T}$ Tauri star LH 332-20, from the catalogue compiled by Herbig \& Rao (1972). It lies in an anonymous dark nebula at unknown distance. It is known that $\mathrm{T}$ Tauri stars may exhibit X-ray flaring activity. In fact, Montmerle et al. (1983) reported a "superflare" in another T Tauri star: ROX-20, in the $\rho$ Oph Cloud Complex. It reached a peak luminosity $L_{x}=1.110^{32} \mathrm{erg} \mathrm{s}^{-1}(0.3-2.5 \mathrm{keV})$, and an integrated flare X-ray energy $E=10^{34}$ erg s $^{-1}$. We propose the T Tauri star LH 332-20 as the most likely candidate for GRS 1100-77.
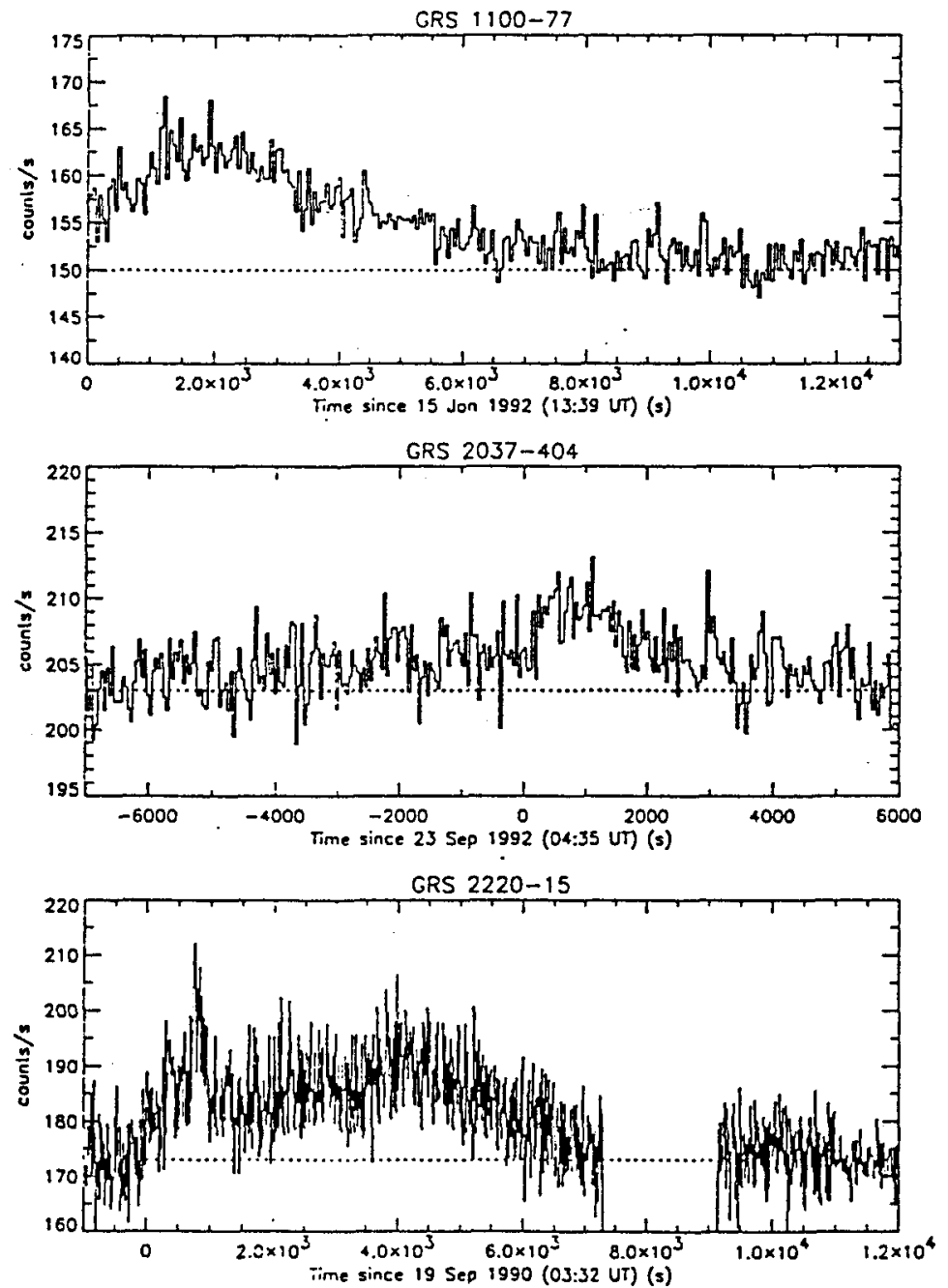

Fig. 1. X-ray light curves in the 8-15 keV energy band of the three fast $\mathrm{X}$-ray transients discovered with WATCH on GRANAT. 
GRS 2037-40. The error box includes the Mira star U Mic. On a plate taken at our request six days after the discovery, this star was near maximum brighteness at $7^{\mathrm{m}} 0$. However we do not consider this star as a candidate due to the different time scales of the involved physical processes. Hudec (priv. comm.) proposed another candidate inside the WATCH error box to be further investigated: the variable star RV Mic, discovered by C. Hoffmeister in 1948. Although it is classified as a Mira star, it is apparently a highly variable object. Nevertheless, we carefully examined the Schmidt plate and found no object inside the WATCH error box that differed by more than $0^{\mathrm{m}} 5$ compared with the corresponding ESO Sky Survey plate.

GRS 2220-15. The high peak flux (2.1 Crab) was similar to the peak of the fast transient at $20^{\mathrm{h}} 14^{\mathrm{m}}+30.9^{\circ}$ discovered with $O S O-8$ in 1977 (Serlemitsos et al. 1979). For GRS 2220-15, $L_{\mathrm{X}}=1.510^{30}(\mathrm{~d} / 1 \mathrm{pc})^{2} \operatorname{erg~s}^{-1}(8-15 \mathrm{keV})$, or $L_{\mathrm{X}}=610^{30}(\mathrm{~d} / 1 \mathrm{pc})^{2} \mathrm{erg} \mathrm{s}^{-1}(2-15 \mathrm{keV})$ if we assume a Crab-like spectrum. Clearly, a dMe or a dKe star may be excluded. A possibility would be to consider a RS CVn system at a distance $\leq 10 \mathrm{pc}$, but none of the two variable stars contained in the error box belong to this class: SS Aqr (a Mira-type star) and BW Aqr (an Algol system). If all the flares that BW Aqr can produce are Algoltype (i.e., the peak luminosity is $\sim 510^{30} \mathrm{erg} \mathrm{s}^{-1}$, White et al. 1980), this star can be discarded as a serious candidate for this event.

Considering the high peak luminosities, a flare of a dMe or a dKe star may be excluded in all the three cases. No nearby RS CVn stars from the list of Lang (1992) were found in the error boxes. It may be possible that these events are associated with old isolated neutron stars accreting interstellar matter with unstable nuclear burning (Ergma \& Tutukov 1980). According to Zdunik et al. (1992), when the accretion rate is higher than $10^{13} \mathrm{~g} \mathrm{~s}^{-1}$, the hydrogen burning triggered by electron capture becomes unstable. As the mass of the accreted envelope should be $10^{23} \mathrm{~g}$, several hundred years will be required for accreting this amount of matter. Assuming that the number of isolated neutron stars with such high rate is $10^{5}$ (Blaes \& Madau 1993), one should expect 10-100 fast X-ray transients per year.

\subsection{The longer-duration $\mathrm{X}$-ray transients}

The three longer-duration sources reported in Sect. 2.2. are concentrated near the galactic plane, suggesting that they could be more distant than the three fast X-ray transients described in Sect. 2.1. No nearby dwarf star or RS CVn systems have beed identified in the error boxes. Three variable stars were found inside the GRS 1148-66 error box: CY Mus, UV Mus and TY Mus. In the case of GRS 1624-37, a Schmidt plate was requested on La Silla which was taken on 27 Sep 1992. A previously unknown variable object has been found inside the WATCH error box (Castro-Tirado \& Lund 1995).

In the Ariel $V$ Catalogue of fast X-ray transients, Pye \& McHardy (1983) described $10 \mathrm{X}$-ray transients with durations ranging $0.5-4 \mathrm{~d}$. Some of them were associated with known Be-neutron stars systems, like $4 \mathrm{U} 0114+65$, and 
other with RS CVn systems, like $\sigma$ Cen or DM UMa (for which a flare lasting $1.5 \mathrm{~d}$ was observed by $H E A O-2$ ). The long duration events reported here are likely to have originated from compact objects in both LMXBs and HMXBs.

Acknowledgements: We are very grateful to the staff of the Evpatoria ground station in Ukraine and those of the Lavotchkin and Babakin Space Center in Moscow, where GRANAT was built. Also to G. Pizarro, A. Smette and R. West (ESO) for the Schmidt plates taken at La Silla.

\section{References}

Agrawal P.C., Vaidya G.R., 1988, MNRAS 219, 777

Ambruster C., Snyder W.A., Wood K.S., 1984, ApJ 1984, 270

Ambruster C. et al., 1983, ApJ 269, 779

Ambruster C.W., Wood K.S., 1986, ApJ 311, 258

Blaes O., Madau G., 1993, ApJ 403, 590

Catalano S., 1990, in Active Close Binaries, C. Ibanoglu (ed.), Kluwer, Dordrecht, p. 411

Castro-Tirado A.J., Brandt S., Lund N., 1992, IAU Circ. 5617

Castro-Tirado A.J., 1994, Ph.D. Thesis, Univ. of Copenhagen

Castro-Tirado A.J., Lund N., 1995, in preparation

Connors A., 1988, Ph.D. Thesis, Univ. of Maryland

Cully S.C. et al., 1993, ApJ 414, L49

Ergma E., Tutukov A., 1980, A\&A 84, 125

Gershberg R.A. et al., 1995, these proceedings

Gliese W., 1969, Veröffent. Astron. Rechen-Inst. 22

Heise J. et al., 1975, ApJ 202, L73

Herbig H.G., Rao N., 1972, ApJ 104, 401

Lang K.R., 1992, Astrophysical Data, Springer, Berlin

Lapshov I., Sazonov S., Sunyaev R., 1992, priv. comm.

Lewin W.H.G., Joss P.C., 1981, SSR 28, 3

Linsky J.L., 1991, Mem. S. A. It. 62, 307

Lund N., 1985, in X-ray Instrumentation in Astronomy, J.L. Culhane (ed.), SPIE 597, 95

Montmerle T. et al., 1983, ApJ 269, 182

Pallavicini R., Tagliaferri C., Stella L., 1990, A\&A 228, 403

Pettersen B.R., 1991, Mem. S. A. It. 62(2), 217

Pye J.P., McHardy I.M., 1983, MNRAS 205, 875

Rao A.R., Vahia M.N., 1987, A\&A 188, 109

Rappaport S. et al., 1976, ApJ 206, L139

Schnopper H.W., Delvaille J.P., Epstein A. et al., 1976, ApJ 210, L75

Serlemitsos P., Bunner A., Swank, J.H., 1979, Bull. of the APS 24, 584

Smith M.A. et al., 1993, ApJ 409, L49

Stern R.A., Agrawal P.C., Riegler, G.R., 1981, Nature 290, 573

Swank J.H. et al., 1977, ApJ 212, L73

Van der Woerd H. et al., 1989, A\&A 220, 221

White N.E. et al., 1980, ApJ 239, L69

Zdunik et al., 1992, ApJ 384, 129 